\title{
Dinâmica de relaxação em meios dielétricos: uma aplicação envolvendo osciladores harmônicos
}

\author{
Adriano Dofl' ${ }^{1}$, Jalves S. Figueira e Jean C. Gentilini \\ Universidade Tecnológica Federal do Paraná , Pato Branco, PR, Brasil \\ Recebido em 29/11/2010; Aceito em 10/12/2010; Publicado em 21/3/2011
}

\begin{abstract}
Neste trabalho exploramos o potencial didático de um modelo para descrição da polarização dielétrica que é baseado na dinâmica de um oscilador amortecido. O modelo em questão pode ser utilizado como um exemplo de aplicação de conhecimentos elementares a descrição de sistemas físicos mais complexos. A fim de ilustrar a dinâmica do movimento orientacional de um dipolo elétrico sujeito a ação de forças dissipativas, consideramos a análise do movimento de uma agulha de bússola sujeita a ação de um campo magnético harmônico. Com base nos dados gerados para um conjunto de quatro bússolas, conseguimos reproduzir qualitativamente a principal virtude do modelo que foi descrever os dois máximos de absorção que são observados em algumas substâncias dielétricas.
\end{abstract}

Palavras-chave: osciladores harmônicos amortecidos e forçados, polarização dielétrica, tracker.

In this work we explore the didactic potential of a model for description of dielectric polarization which is based on the dynamics of a damped oscillator. This model can be used as an example of application of the elementar knowledge in the description of complex physical systems. In order to illustrate the dynamics of an electric dipole subject to action of dissipative forces, we consider the analysis of the movement of a compass needle subjected to the action of a harmonic magnetic field. Based on data generated for a set of four compass, we reproduced qualitatively the main virtue of the model that was to describe the two absorption maxima observed in some dielectric substances.

Keywords: damped and forced harmonic oscillators, dielectric polarization, tracker.

\section{Introdução}

Fenômenos oscilatórios são de extremo interesse em todos os campos da física e o tópico associado a oscilações em particular é um assunto visto na maioria dos cursos introdutórios de física básica. Tradicionalmente, após a introdução dos conceitos de oscilador harmônico e oscilador harmônico amortecido, passa-se então a análise do oscilador harmônico amortecido e forçado. A título de exemplificação de sistemas físicos que são governados por uma equação do tipo oscilador harmônico amortecido e forçado, o circuito RLC submetido a uma tensão periódica usualmente é o exemplo considerado.

Neste trabalho exploramos um modelo para descrição da polarização de alguns meios dielétricos que é justamente baseado na dinâmica de um oscilador amortecido sujeito a ação de uma força harmônica. Este modelo em particular, pode ser utilizado como um exemplo da aplicabilidade de conceitos vistos nos cursos mais elementares a descrição de sistemas físicos mais

\footnotetext{
${ }^{1}$ E-mail: agomes@utfpr.edu.br.

complexos, além de servir como um exemplo complementar aos convencionalmente apresentados nos cursos elementares. A fim de introduzir os conceitos necessários para a compreensão dos principais aspectos do modelo incluímos primeiramente uma seção de revisão dedicada a dinâmica de osciladores amortecidos sujeitos a ação de uma força harmônica restauradora.

Sendo a equação responsável pela dinâmica do oscilador harmônico amortecido e forçado uma equação diferencial de segunda ordem, na maioria dos textos o tratamento desta é feito considerando as técnicas do cálculo diferencial e integral. Devido ao caráter didático deste trabalho, decidimos em optar por uma abordagem mais simplificada para a obtenção da solução desta equação diferencial que é então baseada na técnica descrita na Ref. [1]. A fim de ilustrar o comportamento de um oscilador harmônico amortecido na presença de uma força externa na seção 3 apresentamos um exemplo bastante peculiar que é baseado no comportamento oscilatório da agulha de uma bússola sujeita a ação de 
um campo magnético harmônico.

Com o objetivo de trazer o tema associado ao processo de relaxação dipolar, na seção 4 apresentamos o modelo de Debye [2] para a polarização. O movimento da agulha da bússola sob o efeito da ação de um campo magnético harmônico pode ser utilizado para ilustrar o movimento orientacional de um dipolo elétrico sujeito a ação de forças dissipativas, desta forma aproveitamos os tópicos abordados nas seções 2 e 3 para promover a interpretação dos parâmetros que surgem na equação de Debye. Na seção 5, com o objetivo de ilustrar a aplicabilidade da utilização das idéias associadas as oscilações harmônicas forçadas a descrição de sistemas mais complexos, apresentamos os aspectos gerais do modelo descrito na Ref. [3], que justamente assume uma descrição baseada na dinâmica de um oscilador harmônico amortecido para o comportamento da polarização orientacional de algumas substâncias que apresentam propriedades dielétricas. Finalmente, na seção 6 apresentamos nossas conclusões.

\section{O oscilador amortecido e forçado re- visitado}

Os fenômenos oscilatórios são de extremo interesse em todos os campos da física e o tópico associado a oscilações em particular é um assunto visto na maior parte dos cursos introdutórios de física básica. Abaixo escrevemos a equação de movimento para um oscilador harmônico amortecido sujeito a uma força externa dependente do tempo que denotamos por $F(t)$

$$
\ddot{x}+\gamma \dot{x}+\omega_{0}^{2} x=\frac{F(t)}{m},
$$

onde identificamos $\gamma=\frac{b}{m}, \omega_{0}^{2}=\frac{k}{m}$, sendo $\omega_{0}$ a frequência natural de oscilação do sistema e $b$ o coeficiente de amortecimento. A solução geral desta equação pode ser escrita com uma combinação linear formada pela solução homogênea $x_{h}(t)$, obtida assumindo $F(t)=0$, e a solução particular $x(t)_{p}$. Considerando a força externa $F(t)$ como sendo harmônica, ou seja supondo $F(t)=F_{o} \cos (\omega t)$, podemos obter uma forma para solução particular considerando o método empregado por Feynman [1], ou seja iremos substituir na última expressão $x(t)$ pelo equivalente complexo $z(t)$, sendo então a solução da equação particular identificada como $x_{p}(t)=\operatorname{Re}[z(t)]$. Neste caso, podemos escrever $F(t)=F_{o} \exp (i \omega t)$, e a Eq. (1) pode ser reescrita na seguinte forma

$$
\ddot{z}+\gamma \dot{z}+\omega_{0}^{2} z=\frac{F_{0}}{m} e^{i \omega t} .
$$

Propondo uma solução na forma $z(t)=z_{0} e^{i \omega t}$, após substituir na equação acima, obtemos

$$
z_{0}=\left(\frac{F_{0}}{m}\right) \frac{1}{\left(\omega_{0}^{2}-\omega^{2}+i \omega \gamma\right)}
$$

Portanto, podemos escrever a solução para a Eq. (2) na forma

$$
z(t)=\left(\frac{F_{0}}{m}\right) \frac{e^{i \omega t}}{\left(\omega_{0}^{2}-\omega^{2}+i \omega \gamma\right)} .
$$

A introdução da força harmônica externa $F(t)$ deverá compensar a dissipação devido a atuação da força de atrito, e o sistema deverá oscilar harmonicamente, todavia, devemos esperar que o movimento resultante esteja defasado em relação a força harmônica aplicada ao oscilador, uma vez que os efeitos devido a dissipação resultarão em um atraso do movimento frente a força aplicada.

A constante de fase $(\varphi)$ pode então ser incorporada a solução descrita na Eq. (4), notando que a constante $z_{0}$ pode ser escrita em termos de uma nova constante, que definimos segundo $z_{0}=A e^{i \varphi}$, e que leva a

$$
\begin{aligned}
& A e^{i \varphi}=A(\cos (\varphi)+i \sin (\varphi))=\left[\frac{\left(\omega_{0}^{2}-\omega^{2}\right)}{\left(\left(\omega_{0}^{2}-\omega^{2}\right)^{2}+(\omega \gamma)^{2}\right)}\right. \\
& \left.-i \frac{\omega \gamma}{\left(\left(\omega_{0}^{2}-\omega^{2}\right)^{2}+(\omega \gamma)^{2}\right)}\right]\left(\frac{F_{0}}{m}\right) .
\end{aligned}
$$

Considerando então esta última equação, podemos identificar

$$
\tan (\varphi)=-\frac{\omega \gamma}{\left(\omega_{0}^{2}-\omega^{2}\right)}
$$

A partir da Eq. (5) podemos determinar $\left|z_{0}\right|^{2}=A^{2}$, e o quadrado da amplitude $\left(A^{2}\right)$ assume a forma

$$
A^{2}=\left(\frac{F_{0}}{m}\right)^{2} \frac{1}{\left(\left(\omega_{0}^{2}-\omega^{2}\right)^{2}+(\omega \gamma)^{2}\right)},
$$

e finalmente, a solução particular $x_{p}(t)=\operatorname{Re}[z(t)]$ pode ser escrita como

$$
x_{p}(t)=A(\omega) \cos (\omega t+\varphi(\omega)) .
$$

Na Fig. (1), no painel superior a direita, efetuamos o plot da componente Real de $z_{0}, \operatorname{Re}\left[z_{0}\right]=A \cos (\varphi)$, como uma função da frequência $\omega$, enquanto que no painel central descrevemos o comportamento da componente Imaginária $\operatorname{Im}\left[z_{0}\right]=A \sin (\varphi)$ em termos da frequência. Para obtenção destes gráficos consideramos a seguinte troca de variável $x=\ln (\omega \gamma)$, e atribuímos os seguintes valores para os parâmetros que surgem nestas expressões: $\frac{F_{0}}{m}=1\left[\mathrm{~m} . \mathrm{s}^{-2}\right], \omega_{0}=$ $2\left[\right.$ rad. $\left.s^{-1}\right]$ e $\gamma=2\left[s^{-1}\right]$. 


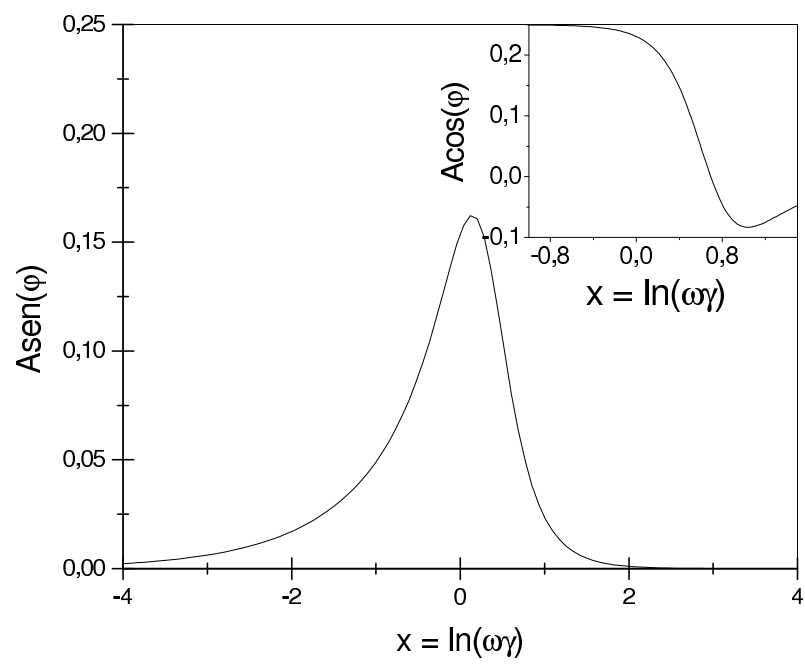

Figura 1 - Componente Real e Imaginária de $z_{0}$ em função da frequência da força harmônica aplicada.

A fim de interpretar os gráficos obtidos, vamos primeiramente efetuar o cálculo da potência média fornecida pela força $F(t)$, que será dissipada no meio onde o nosso oscilador está imerso devido a presença das forças de atrito, ou seja

$$
\begin{aligned}
\overline{P(t)} & =\left\langle\dot{x}_{p}(t) F(t)\right\rangle=\frac{\omega}{2 \pi} \int_{0}^{2 \pi / \omega} \dot{x}_{p}(t) F(t) d t \\
& =-\frac{\omega}{2} A(\omega) F_{0} \sin (\varphi)
\end{aligned}
$$

como $\operatorname{Im}\left[z_{0}\right]=A(\omega) \sin (\varphi)$, podemos escrever a equação descrita acima na forma

$$
\overline{P(t)}=-\frac{\omega}{2} F_{0} \operatorname{Im}\left[z_{0}\right]
$$

que leva a

$$
\overline{P(t)}=\frac{\gamma \omega^{2} F_{0}^{2}}{2 m\left(\left(\omega_{0}^{2}-\omega^{2}\right)^{2}+(\omega \gamma)^{2}\right)} .
$$

O gráfico descrito na Fig. 1, painel central, representa uma figura típica de ressonância, agora se considerarmos a Eq. (10) é possível verificar que a componente imaginária de $z_{0}, A(\omega) \sin (\varphi)$, justamente está associada a dissipação, uma vez que na condição de ressonância $\left(\omega=\omega_{0}\right)$ teremos a taxa máxima de energia sendo transferida para o nosso oscilador, e consequentemente dissipada no meio onde o oscilador está imerso. Em contraste, o quadro exibido no canto superior direito da Fig. 1, nos mostra como se dá a defasagem do movimento em relação a força aplicada, como é possível verificar a partir desta figura para frequências maiores que a frequência de ressonância, $\omega>\omega_{0}$, teremos uma inversão na fase do movimento.

\section{Oscilações amortecidas e forçadas da agulha de uma bússola}

Antes de apresentarmos uma discussão a respeito de algumas idéias associadas com a dinâmica do processo de relaxação em meios dielétricos, iremos considerar nesta seção o comportamento da agulha de uma bússola na presença de um campo magnético harmônico.

$\mathrm{Na}$ Fig. 2 apresentamos uma foto do dispositivo que foi utilizado para aquisição de dados, que consiste de uma bússola convencional disposta em um solenóide. A agulha da bússola se orienta com o campo magnético da terra, designado de $B_{0}$, como é possível notar na figura. O solenóide, orientado perpendicularmente ao campo da terra, é conectado a um gerador de frequências que irá então produzir uma corrente variável em suas espiras que por sua vez criarão no interior do solenóide um campo harmônico do tipo $B(t)=B_{s} \cos (\omega t)$.

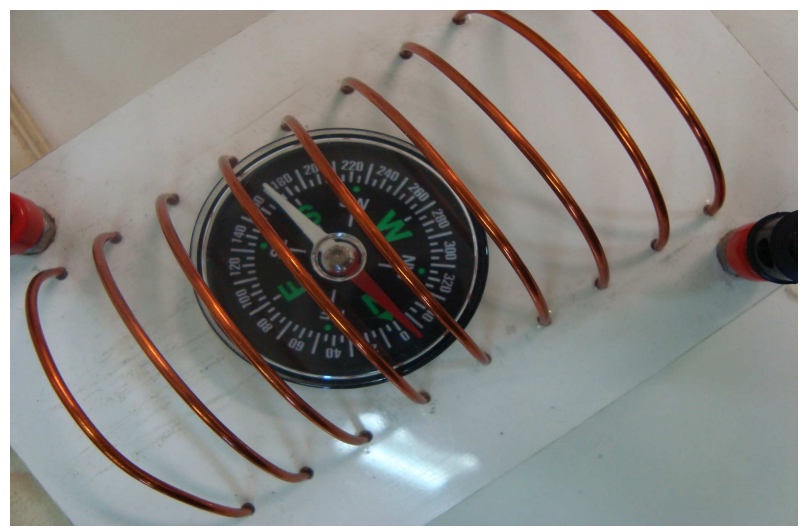

Figura 2 - Dispositivo utilizado para a aquisição de dados.

Assim, quando acionamos o gerador de frequências este faz com que a agulha da bússola passe a oscilar harmonicamente em função do campo $B(t)$ produzido no interior do solenóide. Após o gerador ser desligado a agulha da bússola passa então a diminuir a sua amplitude de oscilação até retornar a posição de equilíbrio, que é caracterizada por sua orientação com os pólos magnéticos da terra na ausência de algum outro campo externo. Na Ref. [4] os autores consideram um arranjo similar ao apresentado neste trabalho para a caracterização de um oscilador amortecido forçado, entretanto, nesta referência são utilizados dois solenóides orientados perpendicularmente, sendo um deles utilizado para gerar um campo constante $B_{0}$, e o outro para gerar o campo $B(t)$. A aquisição de dados nesse caso é realizada considerando um sensor de campo magnético baseado no efeito Hall.

Tendo em vista a proposta didática deste trabalho, adotamos em nosso caso uma abordagem bem mais simplificada e que pode ser facilmente implementada em muitos laboratórios de ensino.

$\mathrm{Na}$ abordagem empregada neste trabalho, para a aquisição de dados consideramos o uso de uma câmera digital convencional para a filmagem do movimento oscilatório de uma dada agulha de bússola quando sujeita a ação do campo harmônico $B(t)$, e efetuamos a análise das imagens geradas considerando o programa Tracker [5], que é um software de domínio publico. 
$\mathrm{Na}$ sequência descreveremos com um certo detalhe quais os procedimentos que foram utilizados para aquisição dos dados, primeiramente fixamos a câmera digital sobre o dispositivo como é possível notar na foto exibida na Fig. 2, na sequência, após a câmera ser acionada ligamos o gerador de frequências que produz uma tensão variável de $(8 \mathrm{~V})$ nas extremidades da bobina. A frequência considerada foi de $(0,612 \mathrm{~Hz})$, uma vez que neste caso as agulhas das bússolas utilizadas passam então a oscilar com amplitudes próximas a amplitude máxima de oscilação $(-\pi / 2$ à $\pi / 2)$, que é determinada a partir da posição de equilíbrio $\theta_{0}$. Então, logo após a agulha da bússola ter atingido este regime, desligamos o gerador de frequências e continuamos filmando o dispositivo até o movimento da bússola cessar completamente, uma vez que estamos interessados apenas na determinação do tempo de relaxação da agulha após cessado a aplicação do campo $B(t)$.

Com base no filme gerado a análise dos dados é feita da seguinte forma, considerando o software Tracker [5] é possível converter o filme em uma sequência de quadros onde a posição agulha da bússola é caracterizada quadro a quadro por um ponto $P=P(\theta, t)$ situado na extremidade da agulha da bússola. Se considerarmos a analogia existente entre o movimento circular e o movimento harmônico podemos aplicar diretamente a Eq. (1) para a descrição deste movimento.

A partir do número de quadros gerados no intervalo de tempo de duração do filme , o programa produz uma tabela de dados contendo a posição angular $\theta_{i}$ no instante $t_{i}$ atribuídos a um dado quadro $(i)$, que então caracteriza a posição da agulha da bússola. Na Fig. 3, exibimos o gráfico gerado a partir do filme obtido para bússola 1, que está representada na Fig. 2. Por conveniência nesta figura excluímos o intervalo de tempo em que a bússola ainda estava na posição de equilíbrio, $\theta_{0}$, devido ao delay gerado entre ligar a câmera e o gerador de sinais.

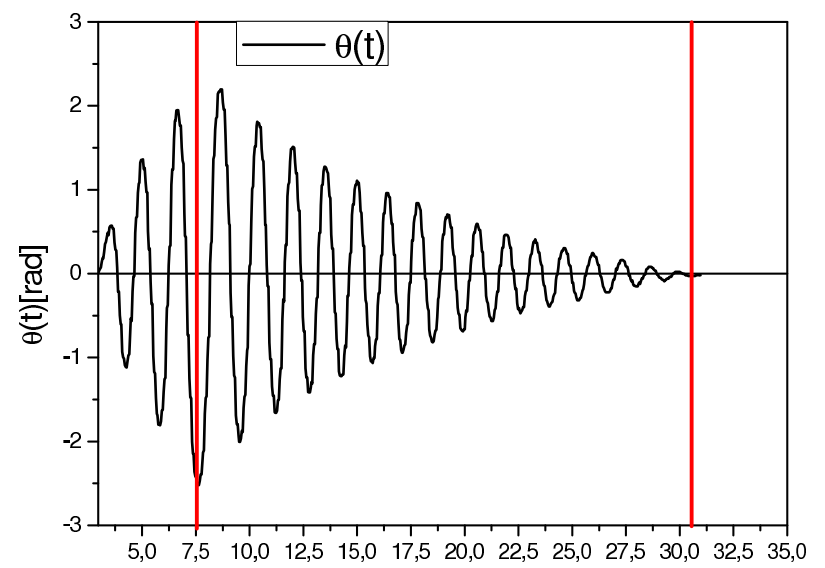

$\mathrm{t}[\mathrm{s}]$

Figura 3 - Tempo de relaxação associado ao conjunto de quatro bússolas.
Como é possível notar na figura, após aproximadamente $7,5 \mathrm{~s}$ a agulha atinge o pico de absorção, e neste momento o gerador é então desligado e a agulha passa então ao regime de relaxação cessando o seu movimento após aproximadamente $30.2 \mathrm{~s}$ do início da gravação. Na Fig. 4, apresentamos os resultados obtidos para quatro bússolas similares, de mesmas proporções e visualmente idênticas, porém, como é possível notar o tempo de relaxação obtido para as quatro bússolas não é o mesmo uma vez que provavelmente o atrito associado ao movimento de cada uma sobre seu eixo de rotação não é o mesmo. Retornaremos a este ponto na seção 5 , por hora, na seção seguinte iremos apresentar resumidamente as idéias essenciais a respeito da descrição da polarização em meios dielétricos considerando a teoria de Debye, uma vez que esta é a mais simples e ainda se aplica a uma grande variedade de materiais dielétricos.

\section{A teoria de Debye e a polarização de meios dielétricos}

No livro Polar Molecules [2], 2 Debye propõe que na ausência de um campo aplicado, o processo de relaxação dielétrica é governado por uma equação do tipo

$$
\dot{P}+\frac{P}{\tau}=0
$$

onde $\tau$ é o tempo de relaxação característico dipolar. $\mathrm{Na}$ presença de um campo elétrico harmônico externo $E(t)=E_{0} \cos (\omega t)$, o processo de interesse deixa de ser a relaxação e teremos uma polarização induzida, que será descrita por

$$
\dot{P}+\frac{P}{\tau}=\frac{\chi_{0}}{\tau} E_{0} \cos (\omega t)
$$
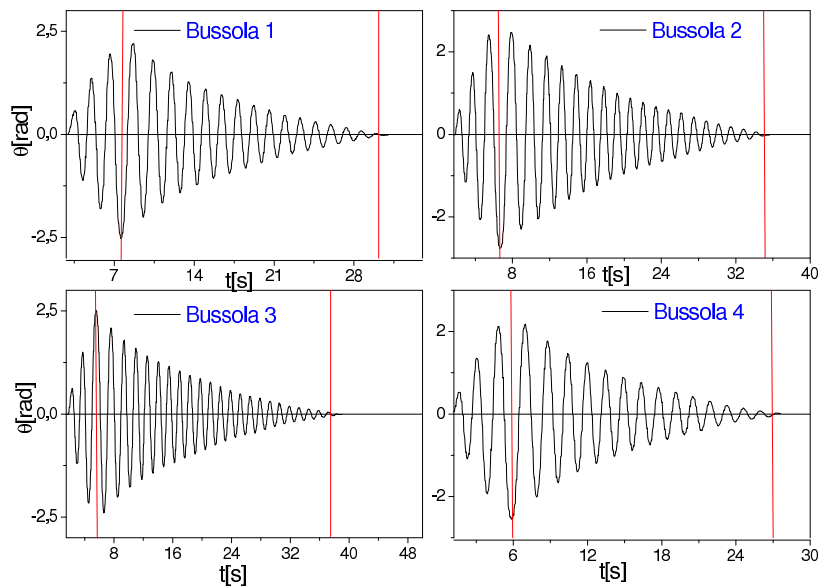

Figura 4 - Tempo de relaxação obtido para a agulha da bússola 1. Nesta figura, a primeira linha vertical delimita o instante de tempo que o gerador é desligado, a segunda linha indica o instante em que o movimento da agulha cessa.

\footnotetext{
${ }^{2}$ Admitindo materiais formados por moléculas contendo um momento de dipolo, e que se encontram muito afastadas(os dipolos não interagem entre si)
} 
onde $\chi_{0}$ é susceptibilidade elétrica na ausência de um campo externo. Como a polarização em termos do campo elétrico aplicado pode ser escrita como $P(t)=$ $\chi E(t)$, e sendo a susceptibilidade $\chi$ em geral expressa em termos das componentes real, $\chi^{\prime}$, e imaginária $\chi^{\prime \prime}$, podemos considerar de forma natural o procedimento utilizado para a resolução da Eq. (1), e portanto podemos aplicá-lo diretamente a Eq. (13), e escrever neste caso

$$
\begin{gathered}
\operatorname{Re}[\chi]=\chi^{\prime}=\frac{\chi_{0}}{\left(1+\tau^{2} \omega^{2}\right)}, \\
\operatorname{Im}[\chi]=\chi^{\prime \prime}=\frac{\tau \chi_{0} \omega}{\left(1+\tau^{2} \omega^{2}\right)},
\end{gathered}
$$

onde usamos o fato que $\chi=\chi(\omega)=\chi^{\prime}(\omega)-i \chi^{\prime \prime}(\omega)$. $\mathrm{Na}$ Fig. 5, plotamos justamente o comportamento das componentes imaginária, $\chi^{\prime \prime}(\omega)$, e real da susceptibilidade $\chi$ para a polarização de um meio dielétrico descrito pela Eq. (13).

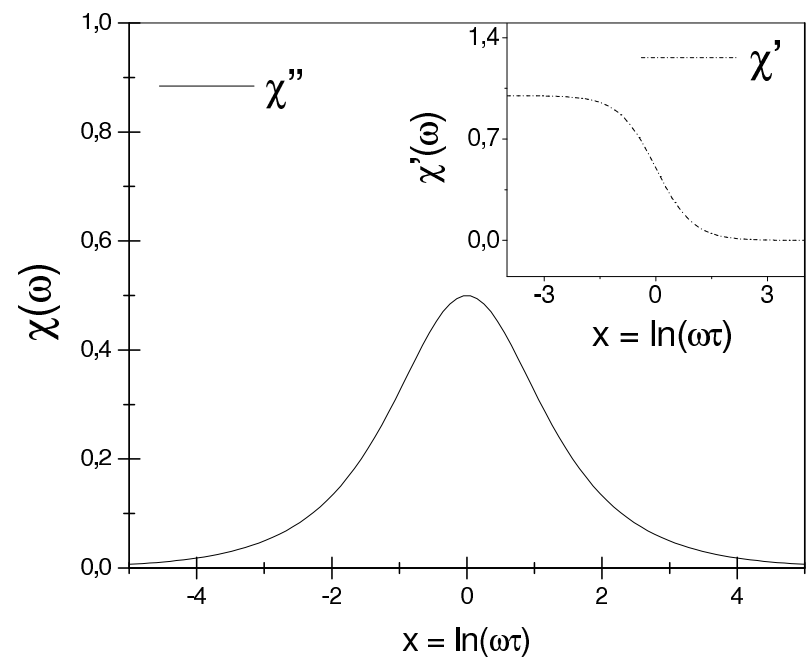

Figura 5 - Dependência do comportamento das componentes $\chi^{\prime}$ e $\chi^{\prime \prime}$ para o modelo de Debye em função da frequência do campo aplicado.

A Eq. (13) é uma equação de primeira ordem, enquanto que a Eq. (1) é uma equação de segunda ordem, que como vimos rege o comportamento de uma agulha de bússola sob a ação de um campo magnético harmônico. Este movimento pode ainda ser visto como o de um dipolo magnético sujeito a ação das mesmas forças atuantes em um oscilador harmônico amortecido e forçado, e se observarmos a similaridade existente entre os gráficos exibidos nas Figs.(1) e (5), poderíamos nos perguntar se não seria então possível descrever a polarização de um dado meio dielétrico assumindo uma equação na forma da Eq. (1).

De fato, no tratamento clássico da interação da radiação com a matéria, uma boa discussão é apresentada na Ref. [6], assume-se uma interação do tipo oscilador harmônico amortecido para a descrição da polarização atômica induzida frente a aplicação de um campo elétrico harmônico, de forma que então é possível determinar neste caso a dependência com a frequência para constante dielétrica do material $\epsilon(\omega)$. A susceptibilidade $\chi(\omega)$ pode ser expressa em termos da constante dielétrica segundo $\epsilon=4 \pi \chi+1$, então com base na discussão apresentada nos dois últimos parágrafos, e aproveitando os resultados descritos na seção 2, podemos portanto efetuar a interpretação do significado físico das componentes $\chi^{\prime}$ e $\chi^{\prime \prime}$ da susceptibilidade.

A componente complexa $\chi^{\prime \prime}$ de fato irá descrever absorção de energia do campo pelo dielétrico, isso pode ser facilmente notado comparando o pico de ressonância descrito na Fig. 1, que como vimos na seção 2 corresponde a situação onde temos a máxima potência sendo transferida pela força externa ao oscilador, com o pico descrito na Fig. 5 que designaremos então de pico de absorção. Por outro lado, a componente real $\chi^{\prime}$, por exemplo, poderá ser usada para caracterização das propriedades óticas do material, como índice de refração $(n)$, uma vez que $n^{2}=\frac{1}{2}\left(\epsilon^{\prime}+\sqrt{\epsilon^{\prime}+\epsilon^{\prime \prime}}\right)$, e notadamente a componente $\chi^{\prime}$ apresenta comportamentos distintos para $\omega<\omega_{0}$ e $\omega>\omega_{0}$.

Por fim, devemos notar que o equivalente da Eq. (6), obtida para a descrição da polarização de um meio

$$
\tan (\varphi)=\frac{\chi^{\prime \prime}}{\chi^{\prime}}
$$

pode então ser utilizado para a caracterização das propriedades dielétricas de um dado material, uma vez que como no caso do oscilador a fase $\varphi$ irá depender das interações dos dipolos com o meio. O modelo de Debye fornece uma descrição adequada para o comportamento da polarização orientacional de muitas substâncias, porém, hoje temos o conhecimento da existência de uma gama de materiais dielétricos que não são caracterizados apenas por um único tempo de relaxação. Desta forma, na seção seguinte iremos justamente ilustrar esta situação considerando um modelo para a polarização de certas substâncias que é então baseado numa equação do tipo oscilador harmônico amortecido forçado.

\section{A dinâmica de relaxação em meios dielétricos: um exemplo envolvendo osciladores harmônicos}

É possível descrever a polarização de um material dielétrico em termos de uma função $\phi_{r}$, denominada de função resposta dielétrica, que reflete então a resposta do meio frente a aplicação de um campo externo $E(t)$ segundo [7]

$$
P(t)=\chi \int_{-\infty}^{t} \phi_{r}\left(t-t^{\prime}\right) E\left(t^{\prime}\right) d t^{\prime} .
$$

A fim de caracterizar as propriedades dielétricas de muitas substâncias, várias funções resposta foram propostas empiricamente no decorrer dos anos, para alguns 
exemplos vide as Refs. [8-12], e para uma apresentação didática remetemos o leitor a Ref. [13]. O comportamento orientacional da polarização de muitos sistemas condensados é bem des-crito por distribuições de tempos de relaxação, e muitos dos resultados obtidos com o uso de funções resposta empíricas hoje são compreendidos neste contexto [7]. Para ilustrar este fato, tendo em vista o apelo didático deste trabalho, iremos então por conveniência assumir a descrição da polarização orientacional que foi apresentada na Ref. [3].

Neste trabalho os autores assumem uma equação do tipo oscilador harmônico amortecido forçado para a descrição da polarização orientacional associado a algumas substâncias, e que reproduzimos abaixo

$$
\ddot{P}+\gamma \dot{P}+\omega_{0}^{2} P=\chi_{0} \omega_{0}^{2} E(t) .
$$

Esta equação pode ser facilmente resolvida empregando o método que apresentamos na seção 2 , de forma que usando novamente a identificação $P(t)=\chi E(t)$, e $E(t)=E_{0} e^{i \omega t}$, temos

$$
\begin{aligned}
\chi^{\prime} & =\frac{\chi_{0} \omega_{0}^{2}\left(\omega_{0}^{2}-\omega^{2}\right)}{\left(\left(\omega_{0}^{2}-\omega^{2}\right)^{2}+(\omega \gamma)^{2}\right)}, \\
\chi^{\prime \prime} & =\frac{\gamma \chi_{0} \omega_{0}^{2} \omega}{\left(\left(\omega_{0}^{2}-\omega^{2}\right)^{2}+(\omega \gamma)^{2}\right)} .
\end{aligned}
$$

Porém, ao invés de considerar apenas uma única constante de amortecimento para todas as moléculas os autores supõem uma distribuição de constantes de amortecimento, que levam as seguintes expressões para $\chi^{\prime}$ e $\chi^{\prime \prime}$

$$
\begin{aligned}
\chi^{\prime} & =\sum_{i} \frac{b_{i} \chi_{0} \omega_{0}^{2}\left(\omega_{0}^{2}-\omega^{2}\right)}{\left(\left(\omega_{0}^{2}-\omega^{2}\right)^{2}+\left(\omega \gamma_{i}\right)^{2}\right)}, \\
\chi^{\prime \prime} & =\sum_{i} \frac{b_{i} \gamma_{i} \chi_{0} \omega_{0}^{2} \omega}{\left(\left(\omega_{0}^{2}-\omega^{2}\right)^{2}+\left(\omega \gamma_{i}\right)^{2}\right)},
\end{aligned}
$$

onde $b_{i}$ é uma constante de normalização. Do nosso exemplo com o oscilador harmônico vimos que $\gamma$ define o tempo de relaxação para um dado oscilador, de forma que a distribuição $\gamma_{i}$ então irá estar associada a uma distribuição de tempos característicos de relaxação. Neste trabalho são assumidas funções de distribuição Gaussianas para as constantes de amortecimento $\gamma_{i}$, e com base nesta descrição os autores conseguem fitar o comportamento de substâncias que exibem dois máximos de absorção em $\chi^{\prime \prime}$, tais como o fluorometano e o clorometano [3].

Como vimos, o movimento da agulha de bússola descrito na seção 3 pode ser visto como o de um dipolo magnético sujeito a ação das mesmas forças atuantes em um oscilador harmônico amortecido e forçado. Devido a analogia que podemos fazer entre os dipolos elétrico $\Leftrightarrow$ magnético, podemos aproveitar os resultados descritos na seção 3 para ilustrar a hipótese aventada na Ref. [3]. Obviamente em nosso caso não poderemos considerar uma distribuição de tempos de relaxação (constantes de amortecimento) devido ao número de medidas que dispomos para as relaxações das quatro bússolas que foram utilizadas, entretanto, podemos ilustrar o efeito de como a suposição de uma distribuição de tempos de relaxação modifica o comportamento da função resposta dielétrica esperado para a polarização de um material que é descrito por este tipo de modelo, assumindo uma média $\langle\tau\rangle$ dos valores obtidos para os tempos de relaxação.

Tabela 1 - Resultados das medidas para $\tau$ obtidas a partir da Fig. 4.

\begin{tabular}{cccc}
\hline bússola & $t_{i}(\mathrm{~s})$ & $t_{f}(\mathrm{~s})$ & $\tau(\mathrm{s})$ \\
\hline 1 & 7,5 & 30,1 & 22,6 \\
2 & 6,6 & 34,8 & 28,2 \\
3 & 5,7 & 37,5 & 31,8 \\
4 & 5,8 & 27 & 21,2 \\
\hline
\end{tabular}

Na Tabela 1, apresentamos os dados obtidos para as quatro bússolas utilizadas e considerando os resultados exibidos nesta tabela efetuamos o plot da componente complexa da susceptibilidade $\chi^{\prime \prime}$, Eq. (20), sendo que assumimos a normalização $\chi_{0} \omega_{0}^{2}=1$ e $\gamma=\frac{a}{\tau}$ e escolhemos o parâmetro $a=45.2$ de forma a obter $\gamma=2$ para a bússola 1.3

O traçado contínuo (em vermelho) exibido na Fig. 6 corresponde a situação onde $\gamma$ é descrito em termos do valor médio $\langle\tau\rangle$ obtido para os tempos de relaxação, como é possível notar abaixo desta linha temos dois picos de absorção de amplitudes próximas que estão ligeiramente deslocadas em relação aos dois máximos de maior amplitude. A partir deste exemplo simples é possível então qualitativamente compreender como a suposição de uma distribuição de tempos de relaxação é então capaz de produzir mais de um máximo de absorção para a componente $\chi^{\prime \prime}$ da susceptibilidade.

A descrição consistente dos mecanismos de polarização e relaxação de meios dielétricos em linhas gerais requer hipóteses a respeito da estrutura microscópica da matéria [14], e uma discussão mais detalhada a respeito deste assunto sairia do escopo deste trabalho, todavia, como mostramos nesta seção é possível compreender os principais aspectos associados a modelos fenomenológicos como o proposto na Ref. [3] assumindo apenas conhecimentos elementares adquiridos pelos alunos ao longo de um curso de Física Básica. Como vimos, o movimento de relaxação da agulha de uma bússola que foi exposta a um campo magnético harmônico $B(t)$ pode ser utilizado para exemplificar o movimento orientacional de um dipolo elétrico na presença de um campo harmônico elétrico $E(t)$. Se imaginarmos em uma dada substância polar os dipolos moleculares distribuídos em domínios, conforme a proposta apresentada na Ref. [14], podemos associar interações distintas

\footnotetext{
${ }^{3}$ Esta normalização em particular foi escolhida com a finalidade da bússola 1 reproduzir as condições do oscilador descrito na seção 2.
} 
entre os dipolos pertencentes a cada domínio, de forma que teremos então um cenário caracterizado por uma distribuição de tempos de relaxação. Ilustramos este efeito considerando um conjunto de quatro bússolas, que como vimos apresentam tempos distintos de relaxação, uma vez que cada uma naturalmente deve estar submetida a diferentes condições de atrito(interações). Finalmente, na situação hipotética de dispormos de um conjunto de $(N)$ bússolas similares a que utilizamos para gerar a Tabela 1, poderíamos enfim vislumbrar o cenário descrito na Ref. [3], onde de fato teríamos uma distribuição de tempos de relaxação e as componentes $\chi^{\prime}$ e $\chi^{\prime \prime}$ seriam então descritas pelas Eqs.(21) e (22).

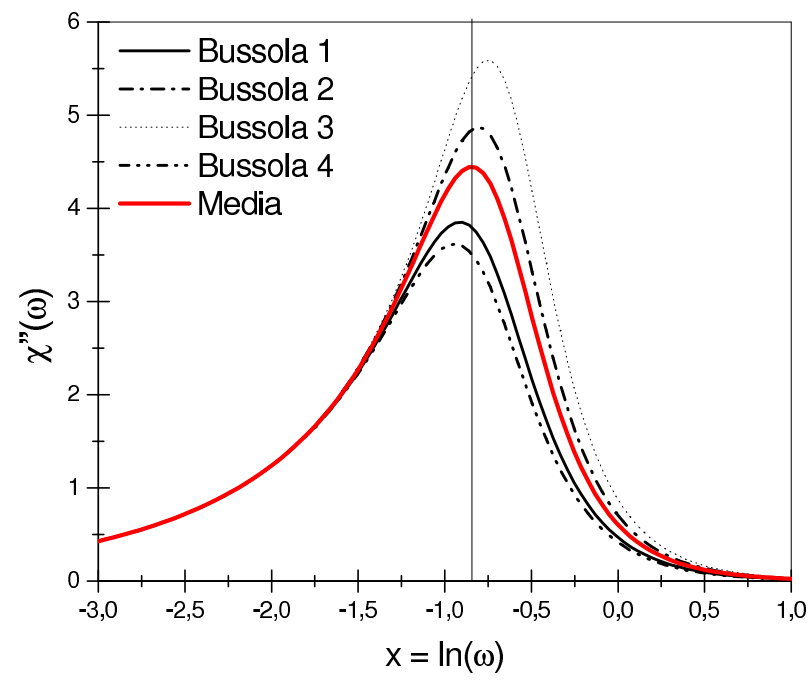

Figura 6 - Comportamento da compomente $\chi^{\prime \prime}$, descrita na Eq. (20), para o conjunto de dados exibido na Tabela 1.

\section{Conclusões}

Como comentamos na seção introdutória, neste trabalho exploramos um modelo para descrição da polarização de alguns meios dielétricos que é baseado na dinâmica de um oscilador amortecido. Considerando a distribuição dos assuntos que apresentamos no texto, podemos então separar as contribuições deste trabalho em duas linhas. No tocante ao ensino de física, como vimos na seção 3 o arranjo da bússola disposta no interior do solenóide (Fig. 2), quando submetida a um campo magnético harmônico, corresponde ao movimento executado por um oscilador amortecido forçado. Um arranjo similar ao considerado neste trabalho já foi proposto na literatura [4]. Entretanto, como salientamos, na Ref. [4] a aquisição de dados é realizada considerando um sensor de campo magnético baseado no efeito Hall, e a análise destes dados é feita considerando um software específico. Tendo em vista a situação atual de muitos laboratórios de ensino de física no país, possivelmente a implementação de um sistema de aquisição e análise de dados como o utilizado na Ref. [4] tornaria inviável a realização deste experimento em muitas instituições ensino.

Desta forma, podemos destacar como nossa principal contribuição a esta linha a abordagem que sugerimos na seção 3 para aquisição e análise dos dados resultantes do movimento oscilatório da agulha da bússola, que como salientamos pode ser facilmente implementada em muitos laboratórios de ensino.

Em relação a aplicação de conhecimentos elementares a descrição de sistemas físicos mais complexos podemos destacar os seguintes pontos. Na seção 4, a fim de trazer o tema associado ao processo de relaxação dipolar, apresentamos o modelo de Debye e aproveitamos as técnicas que introduzimos na seção 2 para a obtenção da solução da Eq. (13). Como vimos o movimento da agulha da bússola sob a ação de um campo magnético harmônico pode ser utilizado para ilustrar o movimento orientacional de um dipolo elétrico sujeito a ação de forças dissipativas, e imerso em um campo elétrico também harmônico. Portanto, considerando os tópicos abordados nas seções 2 e 3 , conseguimos então apresentar o significado físico para as componentes real, $\chi^{\prime}$, e imaginária $\chi^{\prime \prime}$ da susceptibilidade elétrica.

$\mathrm{Na}$ seção 5 descrevemos os aspectos gerais do trabalho [3], que justamente assume um modelo para polarização orientacional que é baseado na dinâmica de um oscilador harmônico amortecido. Como os autores da Ref. [3] argumentam, a principal virtude do modelo proposto foi explicar os dois máximos de absorção que são observados em algumas substâncias dielétricas. Este aspecto do modelo é garantido pela suposição feita pelos autores em assumir uma distribuição de constantes de amortecimento associadas as moléculas que formam uma dada substância.

Considerando os dados coletados para quatro bússolas distintas, que exibimos na Fig. 4, e admitindo que o movimento de relaxação observado para cada agulha de bússola simule o comportamento orientacional de um dipolo elétrico sujeito a ação de forças dissipativas do tipo experimentado por osciladores harmônicos, temos então o gráfico para a componente complexa da susceptibilidade $\chi^{\prime \prime}$ exibido na Fig. 6 para um conjunto de 4 dipolos sujeitos a interações de magnitudes distintas. No contexto da discussão apresentada no último parágrafo da seção 5 , esta situação representaria o caso em que temos quatro dipolos associados a diferentes domínios (regiões) pertencentes a um dado meio dielétrico, de forma que após cessado a aplicação do campo externo, as interações com meio onde os dipolos estão imersos e que fazem com que estes retornem a uma dada configuração de equilíbrio,, 4 são então modeladas pelas forças dissipativas atuantes em um oscilador amortecido.

Conforme comentamos na seção 5, o traçado contínuo vermelho exibido na Fig. 6 corresponde a situação onde consideramos o valor médio para as quatro cons-

\footnotetext{
${ }^{4}$ Admitimos que esta seja caracterizada por uma temperatura de equilíbrio $T_{e}$.
} 
tantes de amortecimento $\left(\bar{\gamma}=\frac{a}{\langle\tau\rangle}\right)$, abaixo desta linha é possível identificar dois picos de absorção de amplitudes próximas que estão ligeiramente deslocados em relação aos dois máximos de maior amplitude.

Admitindo que tivéssemos um número considerável de bússolas com as mesmas características das utilizadas para gerar os dados da Tabela 1, poderíamos extrapolar a situação descrita na Fig. 6 para uma distribuição de constantes de amortecimento de forma que neste limite deveremos nos deparar com dois máximos de absorção formados a frequências distintas, reproduzindo qualitativamente a principal característica do modelo. Na página 123 da Ref. [3], os autores reproduzem o comportamento para $\chi^{\prime \prime}$ obtido para várias substâncias que apresentam dois máximos de absorção no espectro.

O tópico associado a oscilações harmônicas têm sido objeto de muitos trabalhos, apesar do grande volume de artigos que têm abordado o assunto, acreditamos que esta contribuição possa ser de interesse para alunos egressos em cursos elementares de física e também para professores.

\section{Agradecimentos}

O autor A. Doff agradece a Fundação Araucária de Apoio ao Desenvolvimento Científico e Tecnológico pelo apoio financeiro.

\section{Referências}

[1] R.P. Feynman, R.B. Leighton and M. Sands, Lições de Física de Feynman - Edição Definitiva (Editora Book- man, Porto Alegre, 2008 ), v. 1, $1^{\mathrm{a}}$ ed.

[2] P. Debye, Polar Molecules (Claredon Press, Oxford, 1929).

[3] J.J. Makosz and P. Urbanowicz, Z. Naturforsch 57a, 119 (2001)

[4] D. Tomazi e E.C. Caparelli, Revista Brasileira de Ensino de Física 23, 171 (2001).

[5] Douglas Brown, Tracker: Free Video Analysis and Modeling Tool for Physics Education, http://www. cabrillo.edu/ dbrown/tracker

[6] S.M. Rezende, Materiais e Dispositivos Eletrônicos (Editora Livraria da Física, São Paulo, 2004 ), $2^{\text {a }}$ ed.

[7] C.J.F. Böttecher and P. Bordewijk, Theory of Eletric Polarization (Elsevier Science, Amsterdam, 1987) $2^{\mathrm{a}}$ ed.

[8] K.S. Cole and R.H. Cole, J. Chem. Phys. 9, 341 (1941).

[9] R.M. Fuoss and J.G. Kirkwood, J. Am. Chem. Soc. 63, 385 (1941).

[10] R.H. Cole and D.W. Dawidson, J. Chem. Phys. 20, 1389 (1952).

[11] S. Havriliak and S. Negami, J. Polymer Sci. C14, 99 (1966).

[12] G. Williams and D.C. Watts, Trans. Faraday Soc. 66, 80 (1970).

[13] R. Robert, E.L. Kowalski e D.M. Gomes, Revista Brasileira de Ensino de Física 30, 3307 (2008).

[14] L.A. Dissado and R.M. Hill, J. Chem. Soc. Faraday Trans. v2 80, 291 (1984). 